Revista de Psicología de la PUCP. Vol. XVIII, 2, 2000

\title{
Estilos de aprendizaje y aprovechamiento en ingresantes universitarios ${ }^{1}$
}

\author{
Daniel González Lomelî \\ Universidad de Sonora
}

\author{
Sandra Castañeda Figueiras ${ }^{3}$ \\ Universidad Nacional Autónoma de México
}

\author{
María de los Angeles Maytorena Noriega ${ }^{4}$ \\ Universidad de Sonora
}

\begin{abstract}
Se presenta un modelo estructural de las valoraciones de 229 estudiantes acerca de sus estrategias de aprendizaje y el efecto en sus calificaciones escolares. Se realizó un análisis de ecuaciones estructurales que utilizó las respuestas a un inventario de estilos de aprendizaje y las calificaciones escolares del primer semestre. El modelo confirmó la presencia de estrategias de adquisición, de administración de recursos de memoria y de procesamiento de información, similar a lo propuesto por Castañeda (1995). Dado que el modelo explicó sólo $7 \%$ del aprovechamiento escolar, se discute la necesidad de modelar la variable dependiente como una variable latente conformada por un conjunto de indicadores que den cuenta cabal del logro escolar. Palabras claves: estrategias, aprendizaje, aprovechamiento, modelo estructural.
\end{abstract}

\section{Learning styles and achievement in first-year university students}

A structural model of students' appraisals about their own learning strategies and the effects of these strategies on their school scores is presented. Self-reports of 229 new first-year-students of a public university were analized using a structural equations method. Participants responded to a learning-style questionnaire, and their final first semester course scores were also recorded. The measurement model confirmed the existence of acquisition, memory resources managing, and information processing strategies, very much alike those proposed by Castañeda (1995). Since the model explained only the $7 \%$ of the school achievement, the modeling of the dependent latent variable, conformed by a set of observed variables, is stressed and debated. Key words: strategies, learning, achievement, structural model.

I Los autores agradecen el apoyo del Dr. Víctor Corral V. en la revisión del presente documento.

2 Profesor del Departamento de Psicología. Obtuvo el grado de Maestro en la Universidad de Sonora. Correo electrónico dgonzalez@kunkaak.psicom.uson.mx.

3 Doctora en la Universidad Nacional Autónoma de México. Profesora titular del Posgrado en la Facultad de Psicología y fundadora de la Sociedad Iberoamericana de Pensamiento y Lenguaje.

4 Profesora de psicología e instructora de los talleres de Aprender a Aprender. Licenciada en psicología por la $\mathbf{U}$. de Sonora. 

El aprendizaje efectivo en situaciones educativas se concibe, en la literatura internacional, como un proceso constructivo, acumulativo, auto-regulado, orientado a la meta, situado, colaborativo e individualmente diferente, en la construcción del conocimiento y del significado (De Corte, 1995 y 1999). Se dice que es constructivo porque los alumnos no son meros recipientes pasivos sino agentes activos que construyen tanto el conocimiento como las habilidades intelectuales requeridas. Es acumulativo porque los aprendices construyen el conocimiento nuevo sobre la base de conocimientos previos factuales, declarativos y procedimentales (incluyendo los metacognitivos). Es auto-regulado porque el alumno es capaz de preparar su propio aprendizaje, como también lo es para tomar los pasos necesario para aprender, para regular su aprendizaje, de proveerse su propia realimentación y juicios sobre estándares de ejecución, así como para mantenerse concentrado y motivado. Es orientado a metas porque provee intencionalidad explícita con conciencia y da orientación interna a las metas. Es situado porque se basa en experiencias de los alumnos en contextos y situaciones auténticos de la vida real, lo que les permitirá utilizar después lo aprendido en las situaciones de trabajo. Es colaborativo porque el aprendizaje es un proceso social, noción central a una concepción constructivista del aprendizaje, donde no sólo se aprenden los conocimientos pertinentes sino también las maneras de pensar, valorar y utilizar las herramientas asociadas a un trabajo determinado, así como para aprender de los demás.

Si bien es cierto que la posición de De Corte es una formulación optimista y muy deseable sobre el aprendizaje efectivo en cualquier ambiente educativo, también es cierto que refleja concepciones sobre el aprendizaje y el aprendiz difícilmente sostenibles a partir de lo que se reporta en la literatura pertinente (Castañeda, Lugo, Pineda y Romero, 1998). Ésta muestra que aun cuando la meta sea deseable, no 
refleja la realidad de los alumnos ni la de su enseñanza en la mayoría de los niveles educativos.

En el campo del aprendizaje estratégico, por otra parte, se establece que los buenos estudiantes no sólo poseen una gran cantidad de conocimiento específico sobre la materia sino, también, lo asocian con estrategias de aprendizaje exitosas adquiridas por experiencias previas. Todas las aproximaciones coinciden en la importancia de fomentar en el alumno procesos de pensamiento y de autorregulación eficientes. Los hábitos estudiantiles sobre búsqueda exitosa de información, mapeo conceptual, formulación de preguntas, elaboración de ideas, razonamiento apropiado, estrategias eficientes de solución de problemas y las referidas a los procesos autorregulatorios son reconocidos como componentes importantes del éxito académico porque permiten a los estudiantes comprender qué es lo que se desea que aprendan y puedan comprometerse activamente con lo que la escuela les pide. De aquí que el interés por la investigación en aprendizaje estratégico ocupe un lugar preponderante en la literatura contemporánea.

\section{Componentes cognoscitivos y afectivo-motivacionales del aprove- chamiento escolar}

La literatura sustenta evidencias teóricas y empíricas que permiten concebir al aprovechamiento escolar como un comportamiento multicausado, de naturaleza multidimensional. Variables tales como el pensamiento abstracto, las estructuras de conocimiento y las estrategias de aprendizaje, entre otras importantes, son clasificadas como variables cognoscitivas determinantes del aprendizaje escolar (Castañeda y Martínez, 1999; Merino, 1993; Seifert, 1995). También, se reconoce la influencia que ejercen otros procesos y estrategias que disparan, mantienen y controlan la inclinación a aprender, éstas son las variables afectivo-motivacionales, particularmente importantes en la autorregulación del proceso mismo (Boekaerts, 1995; Pintrich, 1998). 
Estilos de aprendizaje y aprovechamiento en ingresantes universitarios

Cano y Justicia (1993) realizaron una extensa investigación sobre la relación entre factores académicos (rendimiento alto/bajo) y las estrategias y estilos de aprendizaje en una muestra de 991 universitarios de cursos iniciales y finales de 10 especialidades en la Universidad de Granada.

Utilizando cuestionarios generados bajo líneas de investigación cuantitativa, que enfatizaron el factor persona, así como investigación cualitativa para evaluar el factor situacional, los autores encontraron que los estudiantes difieren en cuanto al tipo de estrategias y el tipo de motivación mostrada por los grupos. En tanto que los alumnos de alto rendimiento utilizan estrategias de procesamiento profundo, memoria de hechos y alta motivación de logro, los de bajo rendimiento se caracterizan por utilizar procesamiento elaborativo, motivación extrínseca y miedo al fracaso.

Al comparar las estrategias en cuanto a los niveles de estudio encontraron que los estudiantes de los primeros cursos diferían significativamente de una especialidad a otra, tanto en las escalas de estrategias de aprendizaje, como en las de motivación. En cambio, los alumnos de los últimos cursos sólo diferían en las referidas a la motivación. Al parecer, durante la formación académica se desarrollan habilidades y estrategias de aprendizaje asociadas al dominio de conocimiento particular por lo que al final de la formación sólo se encontraron diferencias en cuanto al factor motivacional.

Resultados similares fueron encontrados en 1989 por Castañeda y López en estudiantes mexicanos y por Merino (1993) quien encontró que "casi dos terceras partes de los alumnos que ingresan a las licenciaturas muestran serias dificultades en su capacidad para manejar los conceptos abstractos que son indispensables, no sólo para la asimilación de los conocimientos propios de una disciplina, sino para la reflexión que propicia la elaboración de un concepto de sí mismo, del mundo y de la posición y relación que se desea establecer con éste en el futuro" (pp. 5, 6). 
Estos estudios identifican variables cognitivas, afectivas y motivacionales presentes tanto en estudiantes novatos como avanzados. La carencia de hábitos de estudio eficientes favorece que los estudiantes fallen al enfrentar los requerimientos planteados por gradientes de dificultad creciente en materiales y tareas de aprendizaje, en la medida en la que la formación universitaria los requiera (Castañeda y Martínez, 1999). Así, estilos deficientes de aprendizaje estarán afectando directamente el logro académico, propiciando un aprovechamiento pobre.

Otro aspecto importante del aprendizaje efectivo lo constituyen el conocimiento y las habilidades de autorregulación en los salones de clase. Gracias al conocimiento autorregulatorio y la operación de las habilidades de autorregulación, el estudiante puede satisfacer el requisito de establecer, evaluar, planear y regular si se han cumplido las metas de su aprendizaje, evaluar el grado en el que se han logrado y, si es el caso, establecer la modificación, selección o construcción de las estrategias necesarias para el logro de las metas deseadas (Boekaerts, 1995; Pintrich, 1998).

Diversos autores han señalado que factores motivacionales tales como las creencias sobre la propia competencia y las atribuciones de éxito y fracaso que realizan los estudiantes juegan un papel importante en las tareas académicas. Las ligas positivas entre cogniciones auto-referidas sobre la habilidad, por un lado, y el uso de estrategias cognitivas y metacognitivas, por el otro, han sido demostradas en diversos niveles educativos, desde el elemental (Paris y Oka, 1986) hasta el del bachillerato (Pintrich y DeGroot, 1990) y el universitario (Pintrich, De Groot y García, 1992; Volet, 1991). Las áreas investigadas involucran habilidades representacionales de escritura, lectura y cálculo.

Castañeda (1996) identificó los efectos del interjuego entre componentes cognitivos, afectivo-motivacionales y sociales en una tarea de comprensión de textos en un estudio transcultural México-Holanda. Los resultados identificaron predictores afectivo-motivacionales, 
previos y posteriores a la ejecución de la tarea de comprensión. Los predictores "antes" fueron tres: la competencia subjetiva, el estado emocional y la ansiedad (variables de personalidad asociadas a la situación-tarea). Los principales predictores "posteriores" a la tarea fueron también tres: el esfuerzo reportado y la evaluación de la ejecución (variables relacionadas con atribuciones de controlabilidad) y el estado emocional (variable de personalidad).

La interacción entre nacionalidad (factor social), el nivel de comprensión alto/bajo (factor cognitivo) y la condición antes/después de realizar la tarea (factor afectivo-motivacional) jugó un papel fundamental en la generación diferencial de las valoraciones hechas por los estudiantes sobre sus auto-competencias, sus atribuciones de éxito/fracaso y el nivel de control percibido sobre la situación. Las diferencias culturales en las valoraciones entre mexicanos y holandeses, mostraron ser consistentes tanto para variables de tarea, como para variables de personalidad.

González, Corral, Frías y Miranda (1998) afirman que «los estudios revisados sobre el aprovechamiento escolar y la permanencia en los sistemas escolares refieren una serie de dimensiones relacionadas con estos problemas, como las variables de corte académico, psicológico, familiar y contextual, entre otras. Lo que se destaca aquí es la escasa cantidad de estudios que resumen los resultados en un modelo integral que identifique los predictores del aprovechamiento escolar en los diferentes subsistemas educativos» (p. 170).

Con base en los planteamientos arriba descritos, en este estudio interesó conocer en particular las relaciones estructurales potenciales entre las estrategias cognoscitivas de aprendizaje y las calificaciones obtenidas por los estudiantes en el contexto escolar. En específico, las valoraciones que ellos hacen sobre su pericia para aplicarlas. Para lograr el objetivo propuesto, se consideró útil elegir un modelo que hubiera generado desarrollos tecnológicos en el campo de interés en México como fue el caso del modelo integral de enseñanza y aprendizaje estratégicos de Castañeda (1996). 
De este modelo se seleccionó una parte de la porción de evaluación, la del autorreporte sobre estrategias de aprendizaje. En su totalidad, involucra componentes cognitivos, afectivos, motivacionales y sociales para evaluar estrategias de aprendizaje. Dentro de los desarrollos tecnológicos generados en este rubro se encuentra el Inventario de Estilos de Aprendizaje y Orientación Motivacional que consta de 89 reactivos, tipo Likert, organizados en cuatro subescalas que evalúan lo siguiente:

1. Estilos de adquisición de información, compuestos por estrategias de aprendizaje que involucran dos niveles de adquisición:

- las selectivas (o de procesamiento superficial de lo que se está aprendiendo) y

- las generativas (o de procesamiento profundo de la información a ser adquirida).

2. Estilos de recuperación de la información aprendida, constituidos por lo siguiente:

- el estilo de recuperar información ante diferentes tareas académicas $y$

- el estilo de recuperar información durante los exámenes.

3. Estilos de procesamiento de la información, en términos de lo siguiente:

- reproducir la información aprendida (o convergente) y

- crear y pensar críticamente sobre lo aprendido (o divergente).

4. Estilos de autorregulación metacognitiva y metamotivacional, constituidos por tres componentes:

- los del estudiante, en cuanto a su:

$\rightarrow$ Eficacia percibida

$\rightarrow$ Contingencia percibida 
- Autonomía percibida

- Orientación a la aprobación

- los de la tarea de aprendizaje, en términos de:

- Orientación a la tarea en sí

- Orientación al logro

- los de los materiales, en cuanto a su:

- Evaluación

- Regulación

En cada uno de los reactivos, el estudiante proporciona información acerca de la estrategia que utiliza; el esfuerzo que le supone hacerlo; el resultado que produce como medida subjetiva del nivel de habilidad y las creencias, atribuciones y orientaciones motivacionales que se asocian al estudio.

La porción de autorreporte que se utilizó en el estudio mide las valoraciones que los estudiantes realizan sobre la pericia con la que utilizan estrategias de aprendizaje para adquirir, retener, integrar, recuperar y autorregular su aprendizaje. La combinación entre estrategias da lugar a estilos diferenciados para aprender (Castañeda y López, 1989; Castañeda y Martínez, 1999).

Por otra parte, la literatura que fundamenta el modelamiento de relaciones estructurales entre variables observadas y constructos, tema central de esta investigación, plantea la necesidad de poner en juego relaciones directas e indirectas, de manera integral y con carácter confirmatorio, más que exploratorio, la prueba de modelos teóricos que posibiliten la explicación. Borgen (1984) advirtió la importancia de integrar nuestras fragmentadas teorías y aproximaciones metodológicas en una estructura coherente que represente mejor la complejidad de los procesos estudiados. Sugiere, además, dedicar mayor atención a los modelos de causalidad ya que ellos permiten iniciar los procesos de síntesis teórica y empírica. 
Una de esas estructuras es el modelamiento de ecuaciones estructurales (Bentler, 1993). Según Fassinger (1987), un modelo de ecuaciones estructurales completo posee dos componentes: a) un modelo estructural que especifica la estructura causal hipotetizada entre las variables latentes identificadas (construcciones teóricas no observadas directamente) y; b) un modelo de medición que define las relaciones entre las variables directamente observadas y las variables latentes que se estén usando como aproximaciones. Una variable latente de primer orden (por ejemplo, estrategias selectivas o estrategias generativas, en el caso que nos atañe) sería aquella que se construye a partir de indicadores (variables observadas), mientras que una variable de segundo orden (estrategias de adquisición) se modela a partir de constructos o variables de primer orden (Corral, 1995).

Una fortaleza importante del modelamiento de ecuaciones estructurales la constituye el hecho de poder examinar de forma independiente al componente estructural del modelo (el relacionado con la causalidad hipotetizada) del componente de medición. Así, los problemas de medición son analizados desde la perspectiva psicométrica que les corresponde y los problemas estructurales desde la insuficiencia en la investigación teórica.

Otras fortalezas esenciales del modelamiento estructural es su aproximación confirmatoria: (a) derivado del análisis del modelo de medición es posible modificar indicadores y construir categorías nuevas y (b) del análisis del modelo estructural, es posible identificar debilidades teóricas. Ambas ventajas constituyen herramientas útiles para derivar contribuciones importantes que permitan avanzar en la construcción teórica.

Por lo anterior, el propósito del estudio es probar un modelo estructural que permita identificar categorías e indicadores, válidos y confiables, para el diagnóstico de estrategias autorreportadas en estudiantes de licenciatura que expliquen el aprovechamiento escolar. 


\section{Descripción del modelo teórico a probar}

El modelo a probar está constituido por variables latentes de primer y segundo orden (Figura 1).

Se postula que las valoraciones que realizan los estudiantes sobre las estrategias de aprendizaje afectan directamente el aprovechamiento escolar. Las variables latentes de segundo orden que ponen a prueba lo postulado son tres:

1. Adquisición de la información, constituida por las variables latentes de primer orden estrategias de adquisición selectiva y adquisición generativa.

2. Recuperación de la información aprendida, integrada por las variables latentes de primer orden estrategias de recuperación en diversas tareas y recuperación en exámenes.

3. Estilos de procesamiento de la información, construida con las variables latentes de primer orden estrategias de procesamiento convergente y estrategias de procesamiento divergente.

Las variables latentes de primer orden son seis y están constituidas por indicadores o variables observadas como se ejemplifica a continuación:

1. Adquisición selectiva: comprendo el vocabulario y las expresiones técnicas utilizadas en las materias que he cursado.

2. Adquisición generativa: traduzco a mis propias palabras lo que quiero aprender.

3. Recuperación en diversas tareas: para tener una mejor retención de lo que aprendí, elaboro dibujos, diagramas, mapas, esquemas y/o cuadros sinópticos.

4. Recuperación en examen: elaboro preguntas sobre lo que creo va a venir en el examen.

5. Procesamiento convergente: cuando el estudio lo requiere, identifico cuáles son las cosas que producen efectos específicos. 
6. Procesamiento divergente: Cuando estudio, analizo las ideas importantes del material, tratando de encontrar diferentes puntos de vista sobre el tema.

\section{Metodología}

\section{Participantes}

La muestra disponible estuvo conformada de 229 estudiantes de primer semestre de una licenciatura de ciencias sociales (158) y una licenciatura de ciencias químico- biológicas y de la salud (71) de una universidad del noroeste de México. La edad promedio de los alumnos fue de 19 años ( $D E=3.8$ años), $78.6 \%$ eran del sexo femenino y al final del primer semestre obtuvieron un promedio general de 81 de calificación $(\mathrm{DE}=9.9)$, siendo alumnos regulares $71.8 \%$. De un total de 50 créditos los alumnos lograron cubrir 45.6 créditos en promedio. La distribución de los estudiantes según la escuela de procedencia nos indica que el mayor porcentaje $(43.2 \%)$ proviene del subsistema estatal -Cobach y Cecytes- seguido del subsistema federal (Cbtis 26\%) y en tercer lugar aparecen el subsistema particular y las escuelas foráneas a la capital del estado de Sonora con el mismo porcentaje $(15.4 \%)$ cada una de ellas.

\section{Instrumentos}

Se consultaron los archivos escolares para revisar los expedientes de cada uno de los estudiantes de la muestra; la información registrada comprendió el promedio final de cada una de las materias cursadas, el promedio general y el número de créditos aprobados durante el semestre 98-2 y la condición de alumno regular o irregular. Se aplicó la porción de autorreporte del EDAOM que es un instrumento de lápiz y papel que contiene 89 reactivos, en una escala tipo Likert con seis opciones de respuesta. En cada reactivo se le solicita al estudiante que proporcione información acerca del uso de cada estrategia, el 


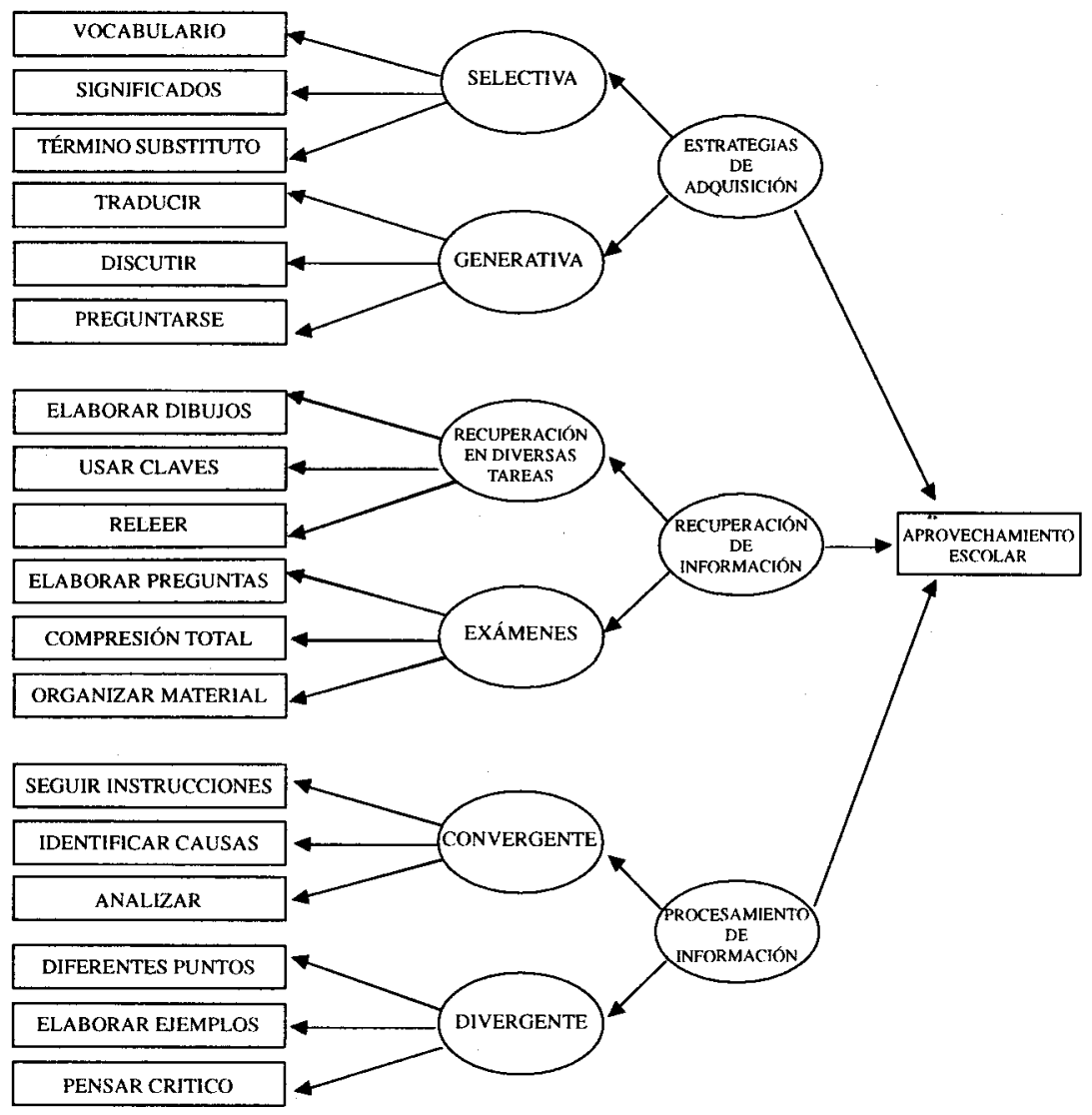

Figura 1. Estructura teórica de estilos de aprendizaje y aprovechamiento escolar. Se presentan tres indicadores por cada variable de primer orden a manera de ejemplo.

esfuerzo que le supone hacerlo y el resultado obtenido. Castañeda y Martínez (1999), han reportado validez de los constructos y consistencia interna de las escalas y de todo el instrumento (alfa de .93). Para este estudio se utilizaron solamente los 40 reactivos que mide estrategias de aprendizaje, desde aquellas que sirven para adquirir la información a ser 
aprendida; las que permiten recuperarla y las que caracterizan los estilos de procesamiento mediante los cuales la información es construida. A continuación, se ejemplifican un par de reactivos de cada subescala:

1. Estilos de adquisición de información:

Adquisición selectiva o superficial (7 reactivos).

- Comprendo el vocabulario y las expresiones técnicas utilizadas en las materias que he cursado.

- Cuando estudio, entiendo el sentido particular de una palabra que tienen varios significados a partir del contexto en el que se encuentra.

Adquisición generativa o profunda (7 reactivos).

- Cuando quiero aprender bien un material de estudio elaboro imágenes mentales que lo representen con claridad.

- Guío mi comprensión y aprendizaje, elaborando ejemplos que relacionen mi propia experiencia con lo que debo aprender.

2. Estilos de recuperación de información:

Recuperación en diversas tareas (6 reactivos).

- Para tener una mejor retención de lo que aprendí, elaboro dibujos, diagramas, mapas, esquemas y/o cuadros sinópticos.

- Para que no se me olvide lo que aprendí, elaboro una imagen mental en mi cabeza que represente lo más importante.

Recuperación en exámenes (6 reactivos).

- Elaboro preguntas sobre lo que creo va a venir en el examen.

- Cuando estudio para preparar un examen, una clase o algo parecido, elaboro cuadros sinópticos, diagramas, mapas conceptuales.

3. Estilos de procesamiento de información:

Procesamiento convergente (7 reactivos).

- Para organizar el conocimiento que estoy aprendiendo, hago cuadros 
sinópticos, esquemas, diagramas y resúmenes que me ayuden a integrar la información más importante.

- Por muy complicadas que sean las instrucciones que debo seguir para resolver un problema, un procedimiento, un método o algo parecido, las puedo seguir.

Procesamiento divergente ( 7 reactivos).

- Cuando estudio, analizo las ideas importantes del material, tratando de encontrar diferentes puntos de vista sobre el mismo tema.

- Cuando el estudio lo requiere, identifico cuáles son las causas que producen efectos especificos.

\section{Procedimiento}

Se aplicó el cuestionario a todos los alumnos de primer ingreso, de manera grupal y voluntaria en sus salones de clase, presentando la tarea como parte de las actividades del programa de servicios estudiantiles de la Universidad. Después se realizaron análisis de frecuencia para las variables descriptivas sociodemográficas de género, estado civil, trabajo, lugar de origen, materias reprobadas y grado de estudio de los padres y medias con sus respectivas desviaciones estándar para edad, promedio general, e ingreso familiar mensual.

Además se realizó un Análisis Factorial Confirmatorio (AFC), y la prueba del modelo incluyó la medición de bondad de ajuste entre el modelo inclusivo y el modelo restringido (o modelo propuesto). El modelo inclusivo refiere una interrelación total de factores y variables observadas y a pesar de que se acepte que ese tipo de relaciones existe aunque sea en forma mínima en la realidad, en ciencia se busca, sobre la base del principio de parsimonia, modelos simples que expliquen lo más posible.

Para contrastar ambos modelos se utilizó el estadígrafo de $\mathrm{X}^{2}$ el cual compara el grado de diferencias entre dos modelos; aquí una $\mathrm{X}^{2}$ alta y significativa refiere que los dos modelos son diferentes, por lo 
cual debemos buscar una $\mathrm{X}^{2}$ no significativa, es decir que su probabilidad asociada sea mayor a 0.05 , de tal manera que nos muestre que el modelo restringido no es diferente del modelo inclusivo, en términos de poder explicativo.

Otros índices de ajuste utilizados son el Índice Bentler-Bonet de Ajuste Normado (IBBAN), el Índice Bentler-Bonet de Ajuste No Normado (IBBANN) y el Índice de Ajuste Comparativo (IAC), incluidos dentro del programa EQS (Bentler, 1993); estos índices producen resultados que van de 0 al 1.0 y se acepta .90 como índice de ajuste adecuado.

Posteriormente se estimaron las correlaciones entre las variables medidas y los factores, y las covarianzas de las variables latentes entre sí, así como de los errores correspondientes a cada factor; se buscó que las relaciones entre variables observadas y las variables latentes correspondientes fueran altas y significativas, con el fin de que la teoría y la validez de constructo convergente de las medidas fueran confirmadas; además se buscó validez de constructo divergente o discriminante, mostrando que las correlaciones entre algunas variables observadas y uno o más factores que no corresponden -según la teoría- con estas variables observadas, fueran menores y tal vez no significativas (Corral, 1995).

\section{Resultados}

\section{Datos sociodemográficos}

Respecto a la variable educación de los padres de familia en el Cuadro 1, podemos observar que se presentan porcentajes similares (52.2\% para los padres y $55.4 \%$ para las madres) en los niveles educativos comprendidos desde la primaria hasta el nivel técnico o comercio, seguido por $26.4 \%$ en el nivel de licenciatura para los padres y $25 \%$ en el nivel de preparatoria para las madres, observándose también 
una proporción similar en los niveles de licenciatura $(9.7 \%)$ y de preparatoria $(7.7 \%)$ para las madres y los padres respectivamente.

Con relación a la distribución ocupacional de los padres existe una relación inversamente proporcional en los diferentes niveles ocupacionales, donde el mayor porcentaje de las madres $(70.5 \%)$ realiza actividades relacionadas con el hogar y sólo $8.5 \%$ se desarrolla en el campo profesional; para los padres la relación encontrada es diferente, ya que el mayor porcentaje $(71.4 \%)$ se ubica como empleado y poco menos de una quinta parte $(22.4 \%)$ se encuentra desarrollando actividades en el terreno profesional.

\section{Cuadro 1}

Proporción de padres de familia por niveles educativos

\begin{tabular}{|lcc|cc|}
\hline & \multicolumn{3}{c|}{ Eadre } & \multicolumn{2}{c|}{ Madre } \\
& Frecuencia & Porcentaje & Frecuencia & Porcentaje \\
\hline Primaria & 44 & 20.0 & 40 & 17.6 \\
Secundaria & 47 & 21.3 & 58 & 25.5 \\
Técnico/comercio & 24 & 10.9 & 28 & 12.3 \\
Preparatoria & 17 & 7.7 & 57 & 25.0 \\
Licenciatura & 58 & 26.4 & 22 & 9.7 \\
Posgrado & 13 & 5.9 & 5 & 2.2 \\
\hline
\end{tabular}

\section{Modelo estructural de estilos de aprendizaje}

El Indicador de confiabilidad para las subescalas de estilos de aprendizaje así como su media estadística se muestran en el Cuadro 2.

El coeficiente de consistencia interna fue de .66 para adquisición selectiva, mientras que su media es de 4.17 , en una escala de uno (baja adquisición selectiva) a seis (alta adquisición selectiva). Para la subescala de adquisición generativa fue de .46 y su media de 3.88 , en una escala que va de uno (baja adquisición generativa) a seis (alta 
adquisición generativa). Para las estrategias de recuperación en diversas tareas fue de .38 con una media de 4.05 , en una escala de uno (baja recuperación en tareas) a seis (alta recuperación en tareas). Para la subescala recuperación en exámenes fue .49 con una media de 4.13, en una escala de uno (baja recuperación en examen) a seis (alta recuperación en examen). Para procesamiento convergente fue de .48 , mientras que su media fue de 3.79 , en una escala de uno (bajo procesamiento convergente) a seis (alto procesamiento convergente) y de .60 para la subescala de procesamiento divergente con una media de 3.38, en una escala que va de uno (bajo procesamiento divergente) a seis (alto procesamiento divergente). Se obtuvo un alfa de Cronbach igual a .85 para la escala total de estilos de aprendizaje.

La Figura 2 muestra la estructura factorial de los estilos de aprendizaje y su relación con el aprovechamiento escolar, construida a partir del autorreporte de estudiantes de primer ingreso.

En cuanto a las variables latentes de primer orden que constituyen el modelo, éstas quedaron integradas de la siguiente manera. El factor de Adquisición Selectiva, quedó integrado por las variables observadas "comprender vocabulario" (.55), "entender significados particulares" (.71) y "entender términos substitutos" (.69).

El factor de Adquisición Generativa se conformó con los indicadores "traducir a tus propias palabras" (.28), "discutir temas de interés" $(.53)$ y "qué habilidades requiero para entender un material" (.50). 
Estilos de aprendizaje y aprovechamiento en ingresantes universitarios

\section{Cuadro 2}

Análisis de confiabilidad de subescalas de estilos de aprendizaje

\begin{tabular}{|lcc|}
\hline \multicolumn{1}{|c|}{ Variable } & Media & Alfa de Cronbach \\
\hline Adquisición Selectiva & $\mathbf{4 . 1 7}$ & $\mathbf{6 6}$ \\
Vocabulario & 4.05 & \\
Significados & 4.38 & \\
Término substituto & 4.09 & $\mathbf{4 6}$ \\
Adquisición Generativa & $\mathbf{3 . 8 8}$ & \\
Traducir & 4.93 & \\
Discutir & 3.62 & \\
Preguntarse & 3.85 & \\
Recuperación en Diversas Tareas & $\mathbf{4 . 0 5}$ & \\
Elaborar dibujos & 3.24 & $\mathbf{3 8}$ \\
Usar claves tipográficas & 4.04 & \\
Releer & 4.89 & \\
Recuperación en exámenes & $\mathbf{4 . 1 3}$ & \\
Elaborar preguntas & 4.14 & \\
Comprensión total & 4.47 & \\
Organizar material & 3.78 & \\
Procesamiento Convergente & $\mathbf{3 . 7 9}$ & \\
Seguir instrucciones & 4.04 & \\
Identificar causas y efectos & 3.27 & \\
Analizar & 3.66 & \\
Interpretar & 4.23 & \\
Procesamiento Divergente & $\mathbf{3 . 3 8}$ & \\
Diferentes puntos & 3.91 & \\
Elaborar ejemplos & 3.61 & \\
Buscar información & 2.20 & \\
Pensamiento crítico & 4.06 & \\
Ampliar conocimientos & $\mathbf{3 . 1 5}$ & \\
\hline
\end{tabular}

Mientras que el factor de Recuperación en Diversas Tareas quedó integrado por los indicadores "para retener lo que aprendí, elaboro dibujos" (.41), "para mejorar la retención, releo"(.50) y "utilizar claves tipográficas" (.39). 
El factor Recuperación en Exámenes lo conforman las variables "elaborar preguntas para el examen" (.33), "estudio hasta lograr una comprensión total del material" (.64) y "cuando preparo mis exámenes, organizo el material que necesito" (.70).

El factor Procesamiento Convergente quedó integrado por los indicadores "seguir instrucciones de procesos" (.40), "identificar causas y efectos" (.41), "analizar, paso a paso, los componentes de una teoría" (.69) e "interpretar situaciones a partir de lo aprendido" (.51).

El constructo Procesamiento Divergente se conformó por los indicadores "encontrar diferentes puntos de vista sobre el mismo tema" (.53), "elaboro mis propias conclusiones y/o ejemplos" (.52), "buscar información para actualizar lo aprendido" (.40), "pensar creativa y críticamente sobre lo aprendido" (.52) y "ampliar el conocimiento sobre un tema" (.53). Todas estas relaciones estructurales fueron significativas a $p<.05$.

Los estilos de aprendizaje se construyeron de las variables latentes de segundo orden Estrategias de Adquisición (.99), Administración de Recursos de Memoria (.84) y Procesamiento de Información (.99), con pesos factoriales arriba de .83. A su vez, las estrategias de adquisición se integran por los constructos adquisición selectiva (.86) y generativa (.99); la variable administración de recursos de memoria se conformó por los constructos recuperación en diversas tareas (.99) y recuperación en exámenes (.96) y; la variable latente procesamiento de información se integró por los constructos procesamiento convergente (.99) y procesamiento divergente (.97).

El Aprovechamiento Escolar posee una $R^{2}=.07$, lo cual significa que las valoraciones sobre los Estilos de Aprendizaje autorreportados por los sujetos que constituyeron la muestra, explican el $7 \%$ de la varianza del aprovechamiento escolar que constituye nuestra variable dependiente de interés. 


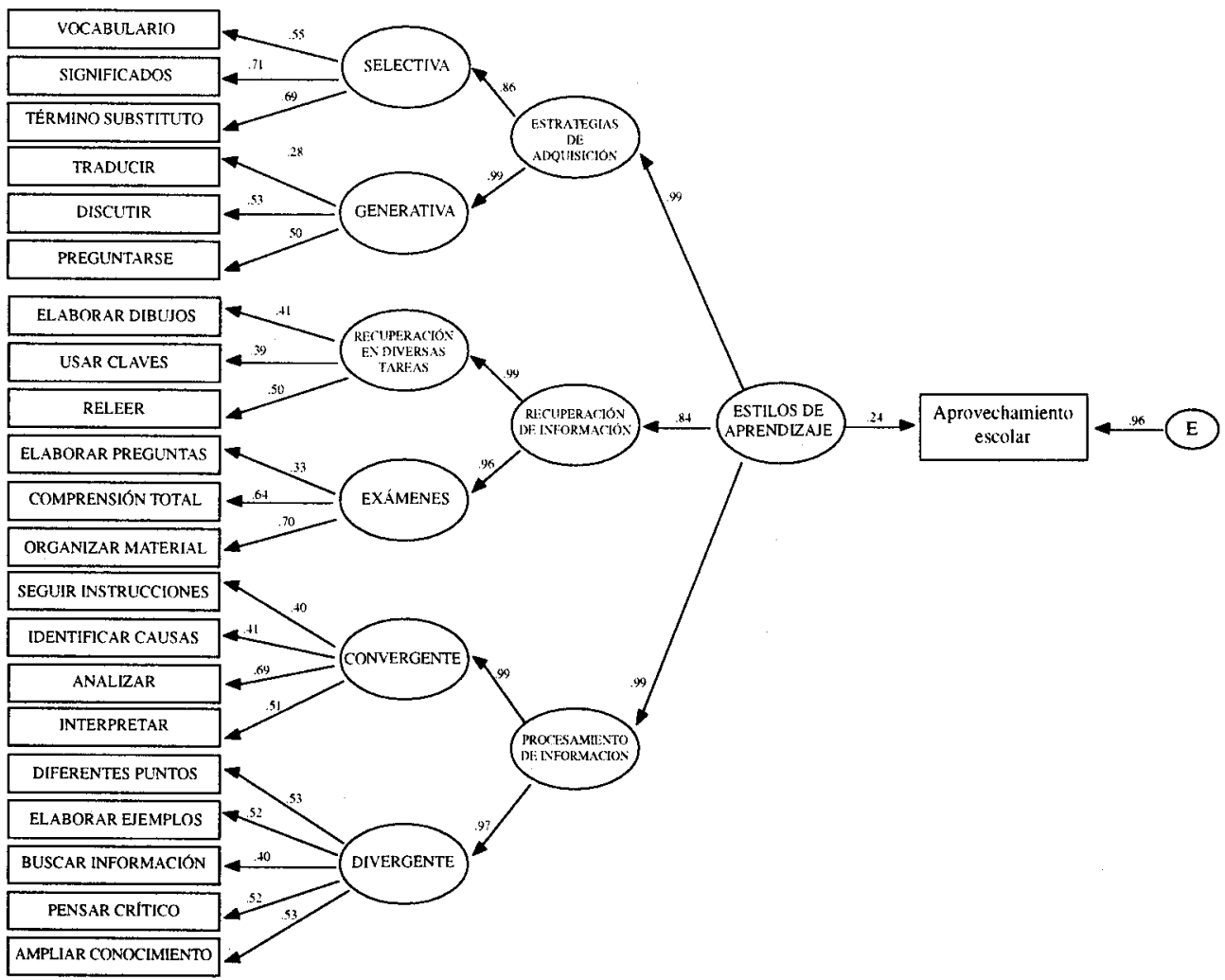
pesos factoriales son significativos a $p<0.05$. $X^{\prime}=201(205 \mathrm{gl}), \mathrm{p}=0.56$; IBAN=0.35, IBANN $=1.0,1 \mathrm{AC}=1.0$. 
Los indicadores de bondad de ajuste muestran que el modelo está respaldado por los datos. La $X^{2}$ resultante fue de 201 (205 gl), asociada a una $p=.56$, el IBBAN es igual a .35 , el IBBANN y el IAC son iguales a 1.0. Esto significa que este modelo teórico, sin ser necesariamente el óptimo, no es significativamente diferente del modelo saturado, en cuanto a poder de explicación.

\section{Discusión}

Es indiscutible que los avances en cognición básica y aplicada al campo educativo han generado modelos cualitativos y derivaciones tecnológicas que intentan explicar el aprendizaje efectivo en los salones de clase. Sin embargo, la realidad de la práctica educativa actual muestra discrepancia entre lo que se conoce sobre los mecanismos que controlan el aprendizaje y el fomento que se debería realizar para lograr aprendizajes de calidad.

Un aspecto que no puede dejarse de lado es el hecho de que en las instituciones educativas mexicanas, todavía no es una actividad curricular cotidiana el modelamiento de las habilidades del pensamiento y mucho menos la evaluación de resultados de aprendizaje, desde un enfoque cognoscitivo.

En este estudio se construyó un modelo estructural de las valoraciones que sobre sus estilos de aprendizaje formularon estudiantes de primer ingreso que resultó similar a la propuesta teórica de Castañeda (1995). La estrategia confirmatoria permitió probar que dicho modelo posee bondad de ajuste tanto estadística como práctica, lo que significa que el modelo posee validez empírica.

Cabe discutir, sin embargo que el poder explicativo mostrado en la relación estructural entre las valoraciones de los estudiantes sobre sus estrategias de aprendizaje y las calificaciones obtenidas, el porcentaje de varianza explicado del aprovechamiento escolar sólo es de 7\%. Esto puede tener relación con varias causas posibles: 
- El hecho de que las estrategias de estudio autorreportadas por los estudiantes, son estrategias eminentemente selectivas, que sólo les permiten un dominio superficial de los contenidos, mas que ser estrategias generativas capaces de ayudarlos en la elaboración y organización del conocimiento nuevo.

- El formato de respuesta utilizado le pide al alumno responder a cada reactivo de manera simultánea, en función de la valoración a tres aspectos: (a) si utiliza la estrategia o no; (b) la dificultad que le representa aplicar la estrategia y (c) el resultado logrado al emplearla. Este arreglo podría estar confundiendo a los sujetos al pedirles que realicen más de una valoración concurrente sobre cada reactivo del cuestionario.

- Las calificaciones escolares utilizadas posiblemente no representen estimados confiables sobre el logro de aprendizajes efectivos, tal y como se plantea en los fundamentos que subyacen al modelo de aprendizaje estratégico. Concepciones tradicionales de medición de resultados de aprendizaje, como son las pruebas de tipo memorístico que miden sólo conocimiento inerte, podrían haber afectado la capacidad predictiva entre la valoración autorreportada sobre la pericia en estrategias de aprendizaje y los indicadores (calificaciones) del aprovechamiento escolar.

Aun cuando en el estudio se realizó una normalización sobre las calificaciones escolares, no se tuvo control sobre la noción de "calidad" que utilizaron los profesores para asignar las calificaciones.

- Variables extra-académicas tales como el apoyo familiar, el locus de control y otras similares, no consideradas en este estudio, y que muy probablemente puedan aportar un porcentaje considerable de explicación de la varianza de la variable dependiente de interés. 
De acuerdo a lo expuesto se puede concluir que con la estructura obtenida en este estudio, la porción de autorreporte del inventar de Estilos de Aprendizaje de Castañeda (1995), mostró que es una escala multifactorial válida para medir las valoraciones que los estudiantes hacen sobre sus estrategias de aprendizaje, en la población de alumnos de la cual se extrajo la muestra del estudio.

En general, podemos afirmar que los estudiantes de la muestra autorreportan un mayor uso de estrategias de adquisición superficial para entrar en contacto con la nueva información a ser aprendida. Así mismo, las estrategias de recuperación autorreportadas reflejan un uso preferencial de estrategias generativas sólo ante la emergencia de exámenes y no ante los requerimientos que deben satisfacer para recuperar información útil para resolver las demandas de tareas de aprendizaje con gradientes de complejidad creciente. Sumado a lo anterior, el estilo de procesamiento autorreportado es de naturaleza convergente.

Algunos factores de confusión que pudieran haber afectado los resultados son los siguientes: (a) el tamaño de la muestra con relación al número de reactivos utilizados apenas cumplió con el criterio mínimo establecido en la literatura; (b) la inclusión en la muestra de alumnos únicamente de dos disciplinas, lo que reduce la validez externa; (c) el formato de respuesta que podría haber introducido un grado de dificultad no esperado y (d) el uso de calificaciones escolares como único indicador del aprovechamiento de los alumnos. Aun cuando se estandarizaron para corregir la diversidad entre profesores, no se controló el criterio utilizado para asignar las calificaciones.

Con el fin de evitar la posible contaminación de los datos provocada por el formato de respuesta presentado a los sujetos, se propone separar la medición de la dificultad que conlleva utilizar las estrategias de aprendizaje, de la medición de los resultados percibidos por los estudiantes. 
Otra posibilidad es utilizar la porción de ejecución del EDAOM, que permita evaluar no nada más la valoración que el estudiante hace sobre su pericia ante cada estrategia de aprendizaje, sino la demostración que ponga a prueba su habilidad en la aplicación de la estrategia misma. El EDAOM incluye tareas orientadoras, puentes de contenido e instrucciones generativas que permiten medir tanto en operante libre, como en operante restringida (por inducción o por imposición) los productos actuales del aprendizaje. También permite medir el desarrollo potencial a partir de contextos de evaluación de zona de desarrollo proximal.

En cuanto a la medición del aprovechamiento escolar es necesario incluir medidas que sean integradas en una variable latente sobre desempeño escolar, que consideren indicadores como materias aprobadas/ reprobadas, estatus regular/irregular de los alumnos, criterios de asignación de calificaciones y otros similares, con el fin de fortalecer el modelo de medición del desempeño de los alumnos y no contar sólo con un índice formado a partir de las calificaciones finales.

La investigación en medición de comportamientos multidimensionales multicausados requiere de estrategias metodológicas que analicen el interjuego entre consideraciones psicológicas y psicométricas, dentro de un contexto de justificación confirmatoria, que ponga a prueba las relaciones estructurales, directas e indirectas, que identifique el poder explicativo de los constructos y minimice la influencia del azar en la interpretación de las evidencias obtenidas.

\section{Referencias}

Bentler, P. M. (1993). EQS: Structural Equations Program Manual. Los Angeles: BMPD Statistical Software.

Boekaerts, M. (1995). The interface between intelligence and personality as determinants of classroom learning. En D. H. Sakloske y M. 
Zeidner (Eds.), International Handbook of Personality and Intelligence (pp. 161-183). NuevaYork: Plenum Press.

Borgen, F. (1984). Counseling psychology. Annual Review of Psychology, 35, 579-604.

Cano, F. y Justicia, F. (1993). Factores académicos, estrategias y estilos de aprendizaje. Revista de Psicología General y Aplicada, 46, 89-99.

Castañeda, S. (1995). El Inventario de Estilos de Aprendizaje y Orientación Motivacional: EDAOM. Reporte de trabajo interno del Depto. de Psicología Educativa del Posgrado de Psicología de la UNAM.

Castañeda, S. (1996). Interfase afectivo-motivacional en la comprensión de textos: estudio transcultural México-Holanda. Revista Latina de Pensamiento y Lenguaje, 4, 169-185.

Castañeda, S. y López, O. M. (1989). La psicología del aprendizaje escolar. En: S. Castañeda y M. López, (Eds.), La psicología cognoscitiva del aprendizaje: aprendiendo a aprender (pp. 2556). México: UNAM.

Castañeda, S., Lugo, E., Pineda, L. y Romero, N. (1998). Evaluación y fomento del desarrollo intelectual en la Enseñanza de Ciencias, Artes y Técnicas: un estado del arte. En S. Castañeda (Ed.), Evaluación y fomento del desarrollo intelectual en la enseñanza de ciencias, artes y técnicas en el umbral del siglo XXI (pp.17-137). México: UNAM, CONACYT-PORRÚA,.

Castañeda, F. S. y Martínez, R. R. (1999). Enseñanza y aprendizaje estratégicos: Modelo integral de evaluación e instrucción. Revista Latina de Pensamiento y Lenguaje, 4, 251-278.

Corral, V. V. (1995). Modelos de variables latentes para la investigación conductual, Acta comportamentalia, 3, 171-190.

De Corte, E. (1995). Fostering cognitive development. A perspective from research on mathematics learning and instruction. Educational Psychologist, 30, 37-46.

De Corte, E. (1999). Una nueva concepción de la enseñanza y el 
aprendizaje para el Siglo XXI . Revista Latina de Pensamiento y Lenguaje, 4, 229-250.

Fassinger, R. (1987). Use of structural equation modeling in couseling. Psychology Research, 34, 425-436.

González, L. D., Corral V. V., Frías A. M. y Miranda J. (1998). Relaciones entre variables de apoyo familiar, esfuerzo académico y rendimiento escolar en estudiantes de secundaria: un modelo estructural. Enseñanza e Investigación en Psicología, 3, 163183.

Merino, C. (1993). Identidad y plan de vida en la adolescencia media y tardía. Revista Perfiles Educativos, 60, 44-48. México: CISEUNAM.

Paris, S.G. y Oka, E. R. (1986). Children's reading strategies, metacognition, and motivation. Development Review, 6, 25-26.

Pintrich, P. R. (1998). El Papel de la Motivación en el Aprendizaje Académico. En S. Castañeda (Ed.), La Evaluación y el Fomento del Desarrollo Intelectual en la Enseñanza de Ciencias, Artes y Técnicas. Perspectiva Internacional en el umbral del Siglo XXI (pp. 229-261). México: UNAM-CONACYT-Porrúa.

Pintrich, P. R. y De Groot, E. V. (1990). Motivational and Self Regulated Learning Components of Classroom Academic Performance. Journal of Educational Psychology, 82, 33-40.

Pintrich, P. R., De Groot, E. A. M. y García, T. (1992, julio). Student Motivation and Self - Regulated Learning in Different Classroom Contexts. Trabajo presentado en el Congreso Internacional de Psicología Científica, Bruselas, Bélgica.

Seifert, T. L. (1995). Academic goals and emotions: a test of two models. The Journal of Psychology, 129, 543-552.

Volet, E. (1991). Modelling and coaching of relevant metacognitive strategies for enhancing university students' learning. Learning and Instruction, 1, 319-336. 